\title{
Enhancement of hole injection using ozone treated Ag nanodots dispersed on indium tin oxide anode for organic light emitting diodes
}

\author{
Jong-Min Moon, Jung-Hyeok Bae, Jin-A Jeong, Soon-Wook Jeong, \\ No-Jin Park, and Han-Ki Kim ${ }^{\mathrm{a})}$ \\ School of Advanced Materials and Systems Engineering, Kumoh National Institute of Technology (KIT), \\ Gumi 730-701, Korea \\ Jae-Wook Kang and Jang-Joo Kim \\ Department of Materials Science and Engineering, Seoul National University, Silim-dong, \\ Seoul 151-741, Korea and Center for Organic Light Emitting Diodes, \\ Seoul National University, Silim-dong, Seoul 151-741, Korea \\ Min-Su Yi \\ Department of Materials Science and Engineering, Sangju National University, Sangju, \\ Gyeongbuk 742-711, Korea,
}

(Received 14 December 2006; accepted 1 March 2007; published online 20 April 2007)

\begin{abstract}
The authors report the enhancement of hole injection using an indium tin oxide (ITO) anode covered with ultraviolet (UV) ozone-treated Ag nanodots for fac tris (2-phenylpyridine) iridium $\operatorname{Ir}(\mathrm{ppy})_{3}$-doped phosphorescent organic light-emitting diodes (OLEDs). X-ray photoelectron spectroscopy and UV-visible spectrometer analysis exhibit that UV-ozone treatment of the $\mathrm{Ag}$ nanodots dispersed on the ITO anode leads to formation of $\mathrm{Ag}_{2} \mathrm{O}$ nanodots with high work function and high transparency. Phosphorescent OLEDs fabricated on the $\mathrm{Ag}_{2} \mathrm{O}$ nanodot-dispersed ITO anode showed a lower turn-on voltage and higher luminescence than those of OLEDs prepared with a commercial ITO anode. It was thought that, as $\mathrm{Ag}$ nanodots changed to $\mathrm{Ag}_{2} \mathrm{O}$ nanodots by UV-ozone treatment, the decrease of the energy barrier height led to the enhancement of hole injection in the phosphorescent OLEDs. (C) 2007 American Institute of Physics.
\end{abstract}

[DOI: $10.1063 / 1.2719153$ ]

Organic light-emitting diodes (OLEDs) have drawn considerable interest due to their extreme thinness, high peak brightness, high dark room contrast, low power consumption, low cost, superviewing ability, and fast response time. Because OLED performance, such as operating voltage and efficiency, is critically affected by carrier injection, it is imperative to develop a high quality anode layer with a low resistance, high transparency, high hole injection efficiency, and high work function. ${ }^{1}$ Although a Sn-doped $\mathrm{In}_{2} \mathrm{O}_{3}$ (ITO) film is typically used in OLEDs as the anode layer, an imperfect work function alignment with a typical hole transport layer (HTL) is one of the disadvantages of the ITO anode. ${ }^{2-4}$ For this reason, alternative anode materials such as $\mathrm{ZnO}$ doped $\mathrm{In}_{2} \mathrm{O}_{3}$, Al-doped $\mathrm{ZnO}, \mathrm{Ni}, \mathrm{Ag}$, and $\mathrm{Pt}$ have been widely investigated as anode layers in OLEDs ${ }^{5-10}$ In particular, Ag metal is employed in top-emitting OLEDs (TOLEDs) as a reflection layer due to its high reflectivity in the visible range. Recently $\mathrm{O}_{2}$ plasma or UV-ozone treated $\mathrm{Ag}$ anodes showed high hole injection efficiencies due to the high work function of $\mathrm{Ag}_{2} \mathrm{O}(\sim 5.3 \mathrm{eV}){ }^{8,9}$ In addition, a self-assembled monolayer modified Ag electrode was reported as an anode layer for TOLEDs. ${ }^{11}$ However, application of a $\mathrm{Ag}$ anode is restricted in the TOLED due to the opaqueness of the $\mathrm{Ag}$ metal layer. Therefore, most research on the Ag metal electrode is focused on the reflective layer in the TOLEDs. ${ }^{11}$ To use the advantages of a $\mathrm{Ag}$ anode, we employed transparent $\mathrm{Ag}$ nanodots dispersed on an ITO anode as the anode material for OLEDs. In GaN-based LEDs, a metal nanodotdispersed electrode has been recognized as a promising

\footnotetext{
a) Author to whom correspondence should be addressed; electronic mail: hkkim@kumoh.ac.kr
}

Ohmic contact structure. Yang et al. reported that Au nanodots on $p$-GaN improved the performance of a GaN LED by formation of a triple-phase junction point between the metal and $p-G a N .{ }^{10}$ Hsu et al. also reported that a Ni-dot embedded ITO anode could reduce the turn-on voltage of OLEDs. ${ }^{12}$ However, Ag nanodots, which are introduced between the organic layer and the ITO layer, have not been investigated in detail for their effects on the performance of OLEDs.

In this work, we report on the characteristics of an UVozone treated Ag nanodot-dispersed ITO anode for enhancing hole injection in phosphorescent OLEDs. The $\mathrm{Ag}$ nanodot-dispersed ITO anode showed identical electrical, optical, and structural properties to those of the bare ITO/ glass sample. In addition, it was found that $\mathrm{Ag}$ nanodots dispersed on ITO film simply changed to $\mathrm{Ag}_{2} \mathrm{O}$ nanodots after UV-ozone treatment for $15 \mathrm{~min}$. Phosphorescent OLEDs fabricated on the $\mathrm{Ag}_{2} \mathrm{O}$ nanodot-dispersed ITO anode showed a lower turn-on voltage and higher luminescence than those of the OLEDs prepared on the bare ITO anode due to high work function of the $\mathrm{Ag}_{2} \mathrm{O}$.

Ag nanodots $(\sim 10 \AA)$ were deposited on a cleaned ITO anode (Asahiglass Fine Techno Co., Ltd.) using an electron beam evaporator without intentional heating of the substrate. The surfaces of the Ag nanodot-dispersed ITO anode and the bare ITO anode were examined by a scanning electron microscope (SEM). To confirm the existence of the Ag element on the ITO anode, an angled $\mathrm{x}$-ray photoelectron spectroscopy (XPS) examination was carried out. The UV/vis transmittance of the bare ITO, the Ag nanodot-dispersed ITO, and the UV-ozone treated Ag nanodot-dispersed ITO anodes were measured over a wavelength range from 220 to 



FIG. 1. Comparison of SEM surface images and XPS wide scan for (a) bare ITO anode films and (b) Ag nanodot-dispersed ITO.

$880 \mathrm{~nm}$. For simplicity, the Ag nanodot-dispersed ITO anode and the UV-ozone treated Ag nanodot-dispersed ITO anode are hereafter referred to as Ag/ITO and UV-ozone treated Ag/ITO, respectively. Before deposition of the organic layers, both the bare ITO and Ag/ITO anodes were UV-ozone treated for $15 \mathrm{~min}$ to remove organic contamination and to increase work function. To investigate the structural properties of the bare ITO and UV-ozone treated Ag/ITO in detail, synchrotron x-ray scattering examinations were performed. After the UV-ozone treatment, both the bare ITO and the Ag/ITO anodes were simultaneously transferred to an organic/metal evaporation system. 40-nm-thick $\quad \alpha$-napthylphenlylbiphenyl $\quad(\alpha$-NPB) and 30-nm-thick 4,4'-bis(9-carbazolyl)-biphenyl doped with 6 wt $\%\left[\operatorname{Ir}(\mathrm{ppy})_{3}\right]$ were used as the HTL and emission layer (EL), respectively. Subsequently, a 10-nm-thick 2,9dimethyl-4,7-diphenyl-1,10-phenanthroline (bathocuproine) layer was grown on the EL layer as a hole-blocking layer. A 40-nm-thick tris(8-hydroxyquinoline) aluminum layer and a $1 \mathrm{~nm} \mathrm{LiF}$ layer were then deposited as an electron transport layer and an electron injection layer, respectively. Finally, a 100-nm-thick Al cathode layer was patterned using a shadow metal mask. Current density-voltage-luminescence $(J-V-L)$ characteristics of the phosphorescent OLEDs fabricated on different anode materials were measured with a Keithley 2400 and a Si photodiode mounted below the OLEDs.

Figure 1 shows the surface SEM images and the XPS wide scan data of the commercial bare ITO anode (reference sample) and Ag/ITO anode. The surface SEM image of the bare ITO anode in Fig. 1(a) exhibits typical crystalline structure with rectangular and square-shaped grains due to (222) and (440) preferred orientations. The XPS wide scan data shows strong $\mathrm{O} 1 s$, In $3 d$, and Sn $3 d$ peaks, which are indicative of a bare ITO anode layer. However, even though a commercial ITO film was used, there is a fairly strong C $1 s$ peak due to the existence of organic contamination on the ITO anode. Figure 1(b) shows the surface SEM image of the $\mathrm{Ag} / \mathrm{ITO}$ anode with XPS wide scan data. It is clearly shown that the $\mathrm{Ag}$ nanodots with sizes of 5-10 $\AA$ are uniformly dispersed on the ITO anode. Without intentional substrate heating during $\mathrm{Ag}$ deposition, the $\mathrm{Ag}$ layer below the thickness of $10 \AA$ exists as a type of $\mathrm{Ag}$ nanodots due to the effect of surface and strain energies of $\mathrm{Ag}$ atoms. The additional $\mathrm{Ag}$
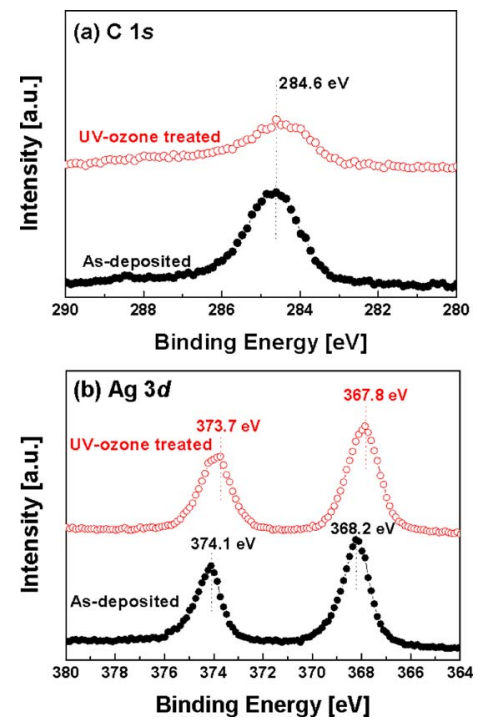

FIG. 2. Change of the core level spectra of (a) C $1 s$ and (b) $\mathrm{Ag} 3 d$ of Ag nanodots after UV-ozone treatment.

$3 d$ peak as well as O $1 s$, In $3 d$, and Sn $3 d$ peaks in the XPS wide scan data shows that the uniformly distributed nanodots on the ITO anode are Ag metal.

Figure 2 shows surface $\mathrm{C} 1 s$ and $\mathrm{Ag} 3 d$ peaks of the $\mathrm{Ag} / \mathrm{ITO}$ anode before and after the UV-ozone treatment. It was noteworthy that the peak intensity of C $1 s$ in Fig. 2(a) was significantly reduced by the UV-ozone treatment. This reduced $\mathrm{C} 1 s$ peak indicates that the UV-ozone treatment effectively removes the organic contamination on the ITO surface as demonstrated in previous reports. ${ }^{13}$ The $\mathrm{Ag} 3 d_{5 / 2}$ core level spectra in Fig. 2(b) exhibit the low binding shift from 368.2 to $367.8 \mathrm{eV}$ after UV-ozone treatment. The low binding shift of the $\mathrm{Ag} 3 d$ core level spectra indicates the transition from $\mathrm{Ag}$ nanodots to $\mathrm{Ag}_{2} \mathrm{O}$ nanodots during UVozone treatment. Chen et al., after investigating a surface modified Ag anode film, reported that room-temperature UVozone treatment of a $\mathrm{Ag}$ anode led to the formation of $\mathrm{Ag}_{2} \mathrm{O}$ on the surface of the Ag anode. ${ }^{7}$ In addition, Waterhouse et al. reported that oxidation of a polycrystalline $\mathrm{Ag}$ foil by ozone treatment led to the low binding energy shift of $\mathrm{Ag}$ $3 d_{3 / 2}$ and $3 d_{5 / 2}$ core level spectra due to the formation of $\mathrm{Ag}_{2} \mathrm{O} .{ }^{14}$ Therefore, it is clear that the UV-ozone treatment is an effective method to remove organic contamination and change $\mathrm{Ag}$ nanodots into $\mathrm{Ag}_{2} \mathrm{O}$ nanodots.

Figure 3 shows the optical transmittance spectra of the bare ITO, Ag/ITO, and UV-ozone treated Ag/ITO anode samples along with an inset showing synchrotron x-ray scat-

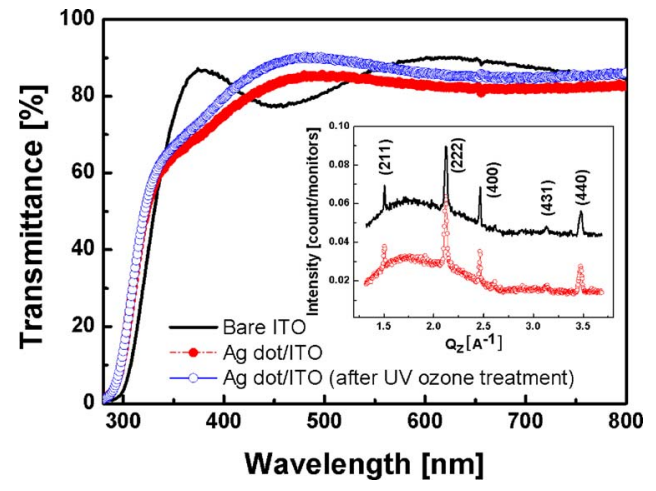

FIG. 3. Comparison of optical transmittance spectra obtained for the bare ITO anode, the $\mathrm{Ag}$ nanodot-dispersed ITO, and the $\mathrm{Ag}_{2} \mathrm{O}$ nanodots dispersed on ITO films with the inset of synchrotron $\mathrm{x}$-ray scattering results. AIP license or copyright, see http://apl.aip.org/apl/copyright.jsp 

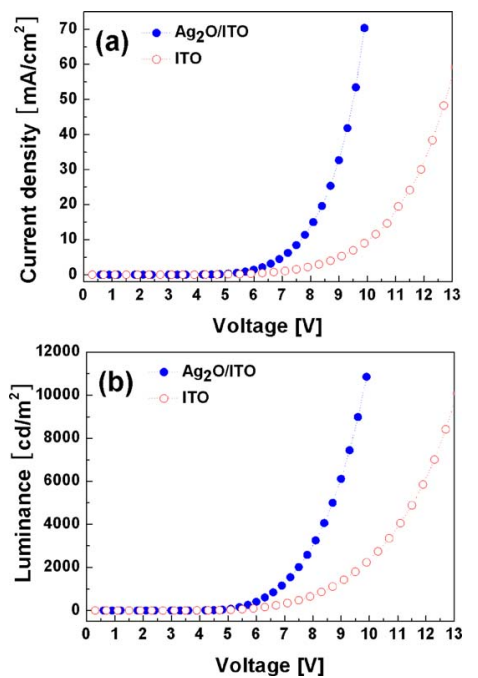

FIG. 4. (a) Current density-voltage and (b) luminance-voltage characteristics of an OLED fabricated on UV-ozone treated Ag nanodot-dispersed ITO and bare ITO anode films.

tering results. It is noteworthy that the transmittance of the as-deposited Ag/ITO anode and UV-ozone treated Ag/ITO anode in the green region is much higher than that of the bare ITO films. Although the transmittance of the asdeposited Ag nanodot-dispersed ITO anode film in the blue region is lower than that of the bare ITO anode films, the average optical transmittance $(\sim 88.9 \%)$ of the Ag nanodotdispersed ITO anode in the green region between 500 and $550 \mathrm{~nm}$ is higher than that of a bare ITO (84.4\%). In addition, it was shown that the transparency of the UV-ozone treated $\mathrm{Ag} / \mathrm{ITO}$ anode is higher than that of the as-deposited $\mathrm{Ag} / \mathrm{ITO}$ anode, due to optical properties of $\mathrm{Ag}_{2} \mathrm{O}$ materials, which have a band gap of $\sim 1.3 \mathrm{eV}$ with the Fermi level range of $4.8-5.1 \mathrm{eV} .{ }^{15}$ Transparency of the $\mathrm{Ag}$ and $\mathrm{Ag}_{2} \mathrm{O}$ nanodots indicates that they can be applied in bottomemitting OLEDs as well as TOLEDs. Using transparent $\mathrm{Ag}$ nanodots dispersed on an ITO anode, we can simultaneously take advantage of both the ITO and the Ag nanodots. The microstructures of the bare ITO and UV-ozone treated Ag/ ITO anodes were compared in the inset of Fig. 3. Due to the small size of the $\mathrm{Ag}_{2} \mathrm{O}$ nanodots, they are not detected using conventional $\mathrm{x}$-ray diffraction methods, so we employed the synchrotron $\mathrm{x}$-ray scattering method. Synchrotron x-ray scattering results of both ITO anodes show identical crystalline peaks at $Q_{z}=1.50$ (211), 2.12 (222), 2.46 (400), 3.13 (431), and 3.47 (440). The dominant orientation of the both ITO anodes was the (222) reflection, and the (400), (431), and (440) reflections were observed as mirror peaks. The identical peaks of both anodes indicate that the microstructure of the $\mathrm{Ag}_{2} \mathrm{O}$ nanodots is amorphous due to the low substrate temperature.

Figure 4 shows the $J-V-L$ characteristics of the OLEDs fabricated on the bare ITO and UV-ozone treated Ag/ITO anode films, respectively. The $J$ - $V$ curve of the OLED fabricated on the UV-ozone treated Ag/ITO anode shows a lower turn-on voltage and a higher current density than those of the OLED fabricated on the reference ITO anode. This indicates that the hole injection from the UV-ozone treated Ag/ITO anode is enhanced by introducing $\mathrm{Ag}_{2} \mathrm{O}$ nanodots between the $\alpha$-NPB and the ITO. The $L-V$ curves of the OLEDs fabricated on the UV-ozone treated Ag/ITO anode and the bare ITO anode films also show a similar steep increase after onset. As expected in the $J$ - $V$ curve, the OLED fabricated on the UV-ozone treated Ag/ITO anode shows a higher luminance than that of an OLED fabricated on the bare ITO anode. The improved $J-V-L$ characteristics of the phosphorescent OLED fabricated on the UV-ozone treated Ag/ITO anode can be explained as follows. It can be related to the high work function of $\mathrm{Ag}_{2} \mathrm{O}$ materials. It has been reported that a $\mathrm{Ag}_{2} \mathrm{O}$ film exhibits $p$-type semiconducting properties and a higher work function $(\sim 5.3 \mathrm{eV})$ than $\mathrm{Ag}(\sim 4.3 \mathrm{eV})$ or ITO $(4.7 \mathrm{eV})$, indicating an ideal anode material. ${ }^{7,9,16}$ Choi et $a l$. also suggested that the work function increased by $0.4 \mathrm{eV}$ when $\mathrm{Ag}$ changed to $\operatorname{Ag}_{x} \mathrm{O}$. ${ }^{9}$ Therefore, uniformly distributed $\mathrm{Ag}_{2} \mathrm{O}$ nanodots lowered the potential barrier between the ITO and the $\alpha$-NPB, enhancing the hole injection. In general, a metal electrode with a high work function such as Pt, Pd, and $\mathrm{Au}$ is unusable as an anode material due to its poor transparency. However, if these metals are fabricated on the ITO anode as a type of nanodot, it is possible to exploit the high work function of a metal anode regardless of its transparency.

In summary, the characteristics of a phosphorescent OLED fabricated on the UV-ozone treated Ag/ITO anode are investigated. It was found that deposition of a $\mathrm{Ag}$ film below the thickness of $10 \AA$ on an ITO anode at room temperature led to the formation of transparent $\mathrm{Ag}$ nanodots. Those $\mathrm{Ag}$ nanodots are changed to $\mathrm{Ag}_{2} \mathrm{O}$ nanodots by conventional UV-ozone treatment. Phosphorescent OLEDs fabricated on an UV-ozone treated Ag/ITO anode showed a lower turn-on voltage and a higher luminescence than those of OLEDs prepared on commercial ITO anodes due to the effect of the high work function of $\mathrm{Ag}_{2} \mathrm{O}$ and effective removal of organic contaminations.

This work was supported by Korea Research Foundation grant funded by Korean Government (MOEHRD: Basic Research Promotion Fund) (KRF-2006-003-D00243) and Ministry of Commerce, Industry, and Energy.

${ }^{1}$ G. Hadziioannou and P. F. van Hutten, Semiconducting Polymers (Willey-VCH, Weinheim, 2000), Chap. 13.

${ }^{2}$ J. Cui, A. Wang, N. L. Edleman, J. Ni, P. Lee, N. R. Armstrong, and T. Marks, Adv. Mater. (Weinheim, Ger.) 13, 1476 (2001).

${ }^{3}$ A. R. Schlatmann, D. W. Floet, A. Hillberer, F. Garten, P. J. M. Smulders, T. M. Klapwijk, and G. Hadziioannou, Appl. Phys. Lett. 69, 1764 (1996).

${ }^{4}$ Y. Park, V. Choong, Y. Gao, B. R. Hsieh, and C. W. Tang, Appl. Phys. Lett. 68, 2699 (1996).

${ }^{5}$ T. J. Marks, J. G. C. Veinot, J. Cui, H. Yan, A. Wang, N. L. Edlemann, J. Ni, Q. Huang, P. Lee, and N. R. Armstrong, Synth. Met. 127, 29 (2002).

${ }^{6}$ H. Kim, C. M. Gilmore, J. S. Horwitz, A. Pique, H. Murata, G. P. Kushto, R. Schlaf, Z. H. Kafafi, and D. B. Dhrisey, Appl. Phys. Lett. 76, 259 (2000).

${ }^{7}$ S.-W. Chen, P.-Y. Hsieh, H.-H. Chiang, C.-L. Lin, H.-M. Wu, and C.-C. Wu, Appl. Phys. Lett. 83, 5127 (2003).

${ }^{8}$ Y. Shen, B. Jacobs, G. G. Malliaras, G. Koley, M. G. Spencer, and A. Ioannidis, Adv. Mater. (Weinheim, Ger.) 13, 1234 (2001).

${ }^{9}$ H. W. Choi, S. Y. Kim, K.-B. Kim, Y.-H. Tak, and J.-L. Lee, Appl. Phys. Lett. 86, 012104 (2005).

${ }^{10}$ J.-L. Yang, J. S. Chen, and S. J. Chang, J. Vac. Sci. Technol. B 23, 2127 (2005).

${ }^{11}$ M.-C. Hung, K.-Y. Wu, Y.-T. Tao, and H.-W. Huang, Appl. Phys. Lett. 89, 203106 (2006).

${ }^{12}$ C.-M. Hsu, C.-L. Tsai, and W.-T. Wu, Appl. Phys. Lett. 88, 083515 (2006).

${ }^{13}$ S. Y. Kim, J.-L. Lee, K.-B. Kim, and Y.-H. Tak, J. Appl. Phys. 95, 2560 (2004).

${ }^{14}$ G. I. N. Waterhouse, G. A. Bowmaker, and J. B. Metson, Appl. Surf. Sci. 183, 191 (2001).

${ }^{15}$ E. Fortin and F. L. Weichman, Phys. Status Solidi 5, 515 (1964).

${ }^{16}$ X. Buwen, S. Yafeng, M. Meng, and L. Chuannan, Microelectron. J. 36, 105 (2005).

AIP license or copyright, see http://apl.aip.org/apl/copyright.jsp 